\title{
Part 6: Rehabilitation and Assistive Robotics
}

\author{
A Custom Serious Games System with Forced- \\ Bimanual Use can Improve Upper Limb Function \\ for Children with Cerebral Palsy - Results from a \\ Randomised Controlled Trial \\ David A. Hobbs ${ }^{\mathrm{a}, \mathrm{b}, *}$, Susan L. Hillier ${ }^{\mathrm{b}}$, Remo N. \\ Russo $^{\mathrm{c}, \mathrm{d}}$ and Karen J. Reynolds ${ }^{\mathrm{a}}$ \\ ${ }^{a}$ Medical Device Research Institute, College of Sci- \\ ence and Engineering, Flinders University, 1284 South \\ Road, Tonsley, SA 5042, Australia \\ ${ }^{\mathrm{b}}$ Division of Health Sciences, University of South Aus- \\ tralia, 108 North Terrace, SA 5001, Australia \\ 'Women's and Children's Health Network, Women's \\ and Children's Hospital, 72 King William Road, North \\ Adelaide, SA 5006, Australia \\ ${ }^{\mathrm{d}}$ College of Medicine and Public Health, Flinders Uni- \\ versity, Sturt Road, Bedford Park, SA 5042, Australia
}

Background: Cerebral palsy (CP) is the most common cause of physical disability in childhood, with the condition affecting both motor and sensory function. Most children with CP favour their dominant upper limb during activities of daily living, resulting in a learned nonuse phenomenon for their non-dominant (ND) limb, and consequently, decreased afferent inputs. The aim of this research was to design, develop, test and trial a custom serious gaming intervention to improve upper limb function, addressing both motor and sensory aspects of the limb, which was novel and had not been attempted before. The hypothesis was that sensory function could be significantly improved through regular use of a home-based haptic gaming system that required coupled bimanual integrated hand use to successfully play the games.

Method: The gaming system was developed using a stage-gate product development process for the hardware and an agile scrum methodology for the software. Universal Design principles, focus groups with children aged 4-16 years old, short-term in-home trials, and a co-design philosophy were adopted to produce the prototype system in 2.5 years. The system, known as $\operatorname{OrbIT}$, is a standalone, self-logging, accessible and haptic serious gaming system that features 15 differ- ent games, which randomise game events to increase player engagement.

Eighteen children with CP (12 male; mean age: $10 \mathrm{yr}$ 8 mo \pm 3 yr 4 mo; Manual Ability Classification System Level: I(2), II(10), III(3), IV(3); CP type: hemiplegia $(n=13)$, diplegia $(n=5))$ trialled OrbIT at home for 6 weeks. The experimental group $(n=$ 10) received afferent haptic vibration to their ND hand via the controller, whereas the haptic feature was disabled for the control group $(n=8)$. Primary outcome measures were tests of sensation (pressure sensitivity, texture discrimination, distal proprioception, and stereognosis) and the Jebsen Taylor Hand Function Test (JFHFT). Assessments were conducted upon enrolment $\left(\mathrm{A}_{1}\right)$, immediately after the intervention $\left(\mathrm{A}_{2}\right)$, and 4 weeks post-intervention $\left(\mathrm{A}_{3}\right)$.

Key results: All 18 children completed the trial, with 2 children withdrawing post-trial. OrbIT was rated highly by families $(7.4 \pm 1.9$ out of 10 , median $=$ $8.0, n=17)$ and overall average system usage was $377 \pm 267$ mins, with no statistical difference in usage between groups $(p=0.07)$. Statistical modelling revealed a significant between group difference for the ND hand for the test of stereognosis between $A_{1}$ and $\mathrm{A}_{2}(p=0.02)$ that did not persist at $\mathrm{A}_{3}(p=0.41)$. When considered as a single cohort $(n=18)$, modelling revealed a strong statistically significant difference for the ND hand for the JTHFT total time ( $p=$ 0.001 ) between $A_{1}$ and $A_{3}$. However, a Type II error cannot be ruled out. A post-hoc analysis revealed an estimated sample size of 55 children for $80 \%$ power.

Conclusion: OrbIT proved to be an acceptable, feasible, and robust technology for children with both unilateral and bilateral CP, when upper limb sensory and motor impairments are present. Sensory improvements were recorded for the intervention group, but did not persist, whereas ND hand function improved significantly for all children post-trial. However, a larger and appropriately powered study needs to be conducted to validate the results of the trial.

Keywords: Serious Games, Universal Design, codesign, cerebral palsy, sensation. 
*Corresponding author. E-mail: david.hobbs@ flinders. edu.au

\author{
Ciranda: A Floor Seat Positioning System and So- \\ cial Enterprise \\ Tulio Maximo ${ }^{\mathrm{a}, *}$ and Erika Foureaux ${ }^{\mathrm{b}}$ \\ a School of Design, The Hong Kong Polytechnic Uni- \\ versity, Jockey Club Innovation Tower, Hung Hom, \\ Kowloon, Hong Kong \\ ${ }^{\mathrm{b}}$ Instituto Noisinho da Silva, Rua Conselheiro Dantas, \\ 183, Belo Horizonte, MG, Brazil
}

Background: One of the first challenges for many children with physical disabilities is to sit independently. A floor seating positioning system enables this milestone, helping the child to maintain eye level with other children, play and learn on the floor, rectify the posture and, as a consequence, helps including the child within its social spectrum. Ciranda is the first comprehensive floor seat solution to attend those needs in Brazil. The design aims to be safe, playful, affordable, and avoid negative connotations commonly seen on floor seat solutions, such as 'tightening' the child with straps or having an appearance of medical equipment. Ciranda design, inspired on cartoons, has an innovative adjustable looping system that hugs the child trunk to the backrest and a set of saddles on the seat that can adjust to different child' body sizes and shapes. The institute Noisinho da Silva, a non-government organisation, designed and produced the Ciranda seat for Brazil market and also developed the Oficina da Ciranda, a social enterprise service endorsed by UNICEF to accommodate the needs of parents in a vulnerable socio-economical situation by teaching them how to build their seat. These multiawarded solutions had benefit more than a thousand children. Now, the institute is studying the applicability of the commercial solution within other markets and the social enterprise within other contexts. This research will focus on structuring the Ciranda project as a model that can be replicated in different regions.

Method: Ciranda and Oficina da Ciranda were developed as a result of a co-design between different stakeholders based on the principle of human-centred design. Anthropometric data were collected from 370 children who cannot sit without support, often children with cerebral palsy, myelomeningocele and multiple disabilities. The families of these children were visited, observed and interviewed. A project requirement was developed using the insights from the data, and a mul- tidisciplinary team of collaborators were formed to codesign the solutions. For the social enterprise service, a model of collaboration was tested in a first workshop in 2007 and improved through the years. This research aims to study and adapt this model aiming to replicate the social enterprise in China and Hong Kong Special Administrative Region.

Key results: Oficina da Ciranda had already benefited more than 6000 people directly. This means that more than 1000 children with disabilities had their floor seating positioning system made by their community and family members, creating a network of people with similar values and struggles to support each other. The Ciranda commercial version was purchased by hundreds of families, contributing to promoting more Oficina da Ciranda workshops with part of the sales profit. Conclusion: Ciranda project connects different stakeholders to form a community that supports each other and to build a solution for one of the many challenges a family with children with physical disabilities has. The model also unravels other challenges common to most assistive technologies, such as access to a device and training for the use and maintenance of the device.

Keywords: floor seat, positioning system, social enterprise, assistive technology service, design.

${ }^{*}$ Corresponding author. E-mail: tperei@polyu.edu.hk, tuliops@gmail.com

\section{Developing an Android Game for Restoring the Motor Functions of Fingers}

Cecilia Sik-Lanyi ${ }^{a, *}$, Patricia Szabo ${ }^{\mathrm{a}}$ and Barbara Bodor $^{\mathrm{a}}$

${ }^{a}$ Department of Electrical Engineering and Information Systems, University of Pannonia, Egyetem u. 10. 8200 Veszprem, Hungary

Background: Each year about 795000 people experience a new or recurrent stroke. The number of the people involved in it is very high, and this number has increased fast. The speed of treatment has a large effect on the person's chance of recovery. The consequence of a stroke is a really serious problem, and it can make one's life harder. This is the reason why this application has been designed; to help people who suffered stroke to heal faster and go back to normal life sooner. There are promising interventions that could be beneficial to improve the aspects of gait including fitness training, high-intensity therapy, and repetitivetask training. Repetitive-task training might also improve transfer functions. Occupational therapy can im- 
prove activities of daily living. Several large trials of rehabilitation practice and of novel therapies are underway to inform future practice. There is a wide range of methods, that can help the patients e.g. serious games for rehabilitation. The idea for developing a new rehabilitation game "Crafted Fingers" has been influenced by a game for children (http://www.fingers-inmotion.de/de/).

Method: We studied the most relevant scientific literatures. Based on the new results and on our earlier experience we have designed a new application for tablet, because we needed bigger surface and because of the touching function it was a good opportunity to combine the technology with the therapy. We used one of the most popular programming languages, the Java and we created the application for android platform. The "Crafted Fingers" application has 5 games: "Piano", "Memory", "Shopping-list", "Fly away" and "Drawing". All games need to be controlled by repetitive movements with fingers on the tablet or on smartphone screen. Every game has different difficulty levels. The "Crafted Fingers" have tested by therapists and elderly people. We measured the scores and time. The results will be shown at the conference.

Key results Playing this game helps everyday tasks become as easy as they were once. The different gestures help differently:

- Tweezer grip: help to pick up small objects,

- Selective finger touch: help in the typing (for example on the phone or on the computer),

- Rotation in the wrist: strengthens the wrist,

- Moving one finger: help the precision,

- Selective finger circles: strengthens the forefinger.

- Selective circles with the thumb: strengthens the thumb.

It will help more patients not just those who suffer from stroke, but those as well who somehow have lost their motoric function in their fingers.

Conclusion: This application will help to restore the motoric function of the fingers for those patients who use it regularly. Even though it will be successful, the patients need the traditional methods as well to achieve the best results during their rehabilitation.

- Benefits: easy to use, exciting, sustaining the attention, improve different skills.

- Disadvantages: It is not enough for the full recovery. It is not fully suitable to strengthen the muscle of the fingers.

Acknowledgements: The authors thank the financial support of Széchenyi 2020 under the EFOP-3.6.1-162016-00015.
Keywords: Android, Game app., Stroke, Rehabilitation, Finger motion.

*Corresponding author. E-mail: lanyi@almos.unipannon.hu

\section{Hand Function in Skills of Modern Day among El- derly Individuals}

Alexandra Danial-Saad ${ }^{\mathrm{a}, *}$, Lorenzo Chiari ${ }^{\mathrm{b}}$, Shlomi Laufer $^{\mathrm{c}}$, Mattia Corzani ${ }^{\mathrm{b}}$ and Michal ElboimGabyzon $^{\mathrm{d}}$

${ }^{a}$ Occupational Therapy Department, Faculty of Social Welfare and Health Sciences, University of Haifa \& The Arab Academic College for Education in Israel, Haifa, Israel

${ }^{\mathrm{b}}$ Department of Electrical, Electronic, and Information Engineering, University of Bologna, Bologna, Italy

${ }^{\mathrm{c}}$ Faculty of Industrial Engineering and Management, Technion Haifa, Israel

${ }^{\mathrm{d}}$ Physical Therapy Department, Faculty of Social Welfare and Health Sciences University of Haifa Haifa, Israel

Background: Elderly individuals in the modern-day environment are obligated to interact with an evergrowing number of technological devices in order to participate in life activities and maintain their independence and quality of life. Operating such devices, such as touchscreens, requires the ability to perform unique motor skills such as tapping, swiping and virtual pinching. Hand function is subject to age-related change. Hence, studying the hand function abilities of the elderly during touchscreen manipulation is an important new research direction. The objective of this study was to characterize elderly hand function performance skills while operating a touchscreen.

Method: The study was a field study conducted in a community center. Study population included 28 community-dwelling elderly individuals (81.9 \pm 4.2 years old) and 16 middle aged $(53.7 \pm 6.2)$ adults. Data collected included subject's characteristics (demographic information, health status). Cognitive status was determined by the Montreal Cognitive Assessment questionnaire. In addition, functional mobility and dynamic balance was assessed by the "Time up and go test". Hand performance was assessed by a) Bilateral strength of hand grip and thumb-finger pinch grips (tip to tip, and three fingers pad to pad) using a calibrated JAMAR hand dynamometer; b) Manual dexterity by Functional Dexterity Test. A software appli- 
cation called Touchscreen-Assessment Tool (TATOO) was used to measure hand performance abilities necessary for operating a touchscreen. Temporal and accuracy measures were collected while performing four of TATOO's tasks. Age effect was examined by comparing the performance of the two groups using the T test. $P \leqslant 0.05$ was considered statistically significant.

Key results: The elderly group were independent in basic and instrumental activities of daily living, presenting high level of functional mobility and dynamic balance as reflected in the TUG scores $(10.3 \pm 3.6)$. However, the MoCA score for the elderly group (21.5 \pm 4.3 ) revealed mild cognitive impairment of the individuals. The middle age group had no cognitive impairment (MoCA score $>30$ ), TUG scores typical to their age $(5.57 \pm 0.95)$, and significantly lower than the elderly group. Significant age effects were observed in the grip strength, three fingers pad to pad pinch strength, and in manual dexterity. In contrast, no age difference was observed in tip to tip pinch strength. Touchscreen operating skills were significantly lower in the elderly, as reflected by the temporal measures and reduced accuracy in the TATOO's task performance. Interestingly, no age difference was observed in the "Pinch ability" task.

Conclusion: Significant differences were found in hand function performance of the elderly when compared to adults as reflected by traditional assessment tools and by TATOO's outcomes. However, the agerelated changes are restricted to pinch skill, as shown in the tip to tip pinch strength and the "Pinch ability" task in TATOO that showed no significant difference between the groups. TATOO demonstrated the potential of becoming an important novel supplement to the hand function assessment toolbox to clinical professionals treating the elderly in the modern world. Future studies with larger samples are warranted in order to establish a normative dataset for the full characterization of hand performance while conducting a variety of touchscreen manipulations.

Keywords: Touchscreen Assessment Tool (TATOO), assessment; hand function, elderly.

*Corresponding author. E-mail: saadalexandra@ gmail.com

Mobile Health Game Development to Motivate Walking for Hematopoietic Stem Cell Transplant Patients

Cecilia Sik-Lanyi ${ }^{\mathrm{a}, *}$, Arpad Kelemen $^{\mathrm{b}}$, Ferenc Revesz $^{\mathrm{a}}$, Csaba Simon ${ }^{\mathrm{a}}$, Shannon Cerbas ${ }^{\mathrm{c}}$, Barbara van De Castle ${ }^{\mathrm{c}}$, Gergo Proszenyak ${ }^{\mathrm{a}}$, Adrian Arvai $^{\mathrm{a}}$ and Yulan Liang ${ }^{\mathrm{b}}$

${ }^{a}$ Department of Electrial Engineering and Information Systems, University of Pannonia, Egyetem u. 10, 8200 Veszprem, Hungary

${ }^{\mathrm{b}}$ University of Maryland, Baltimore 655 W. Lombard St., Baltimore, MD 21201, USA

' Sidney Kimmel Comprehensive Cancer Center at Johns Hopkins Hospital, 800 Orleans St, Baltimore, MD 21287, USA

Background: Fatigue is one of the most commonly reported symptoms for patients going through a hematopoietic stem cell transplant (HSCT) process. Physical activity (PA) has been shown to improve symptoms of fatigue in HSCT patients. The National Comprehensive Cancer Network Clinical Practice Guidelines for Cancer-Related Fatigue advise starting PA slowly and incrementally progressing with distance and time. The use of mobile device applications (apps) promoting fitness may be helpful in increasing PA. Our purpose is to develop a new fitness app called Walking Warrior (WW) for HSCT patients to be used as a step counter and a game.

Method: A game inspired by Candy Crush was ideated and designed as a matching puzzle game with the unique feature of walking to unlock new levels. The WW game play screen includes 10 different tiles, 6 displayed as a cell type, 2 as boosts, and 2 as blocks that make game play more challenging. Cell types include red blood cells, white blood cells, neutrophils, platelets, stem cells, and nerve cells. Each difficulty level has limited number of "moves" a player can make to reach the goal. Each level also has a different goal that the player must reach to get to the next level. The game contains bonuses for more points and obstacles for added challenge and diversity in game play experience. The step counter app tracks steps as the user walks. When the player reached the required number of steps, the game gives them one token and they can continue to play. We developed an admin page with a MySQL database and stored it on a server. Admins and authorized users can check how many tokens the user has and how many steps they made. Evaluating the usability of a game is an essential step in game design and development. Usability of WW and the step counter was assessed by domain experts (4 in game development and 1 in bone marrow transplant nursing). We designed and validated an item-response expert heuristic questionnaire survey/instrument with 40 items for evaluating and assessing usability of WW for 
HSCT patients. Each evaluator rated the questions 1-5, where: $1=$ strongly disagree, $5=$ strongly agree.

Key results: Reliability analysis: Cronbach's alpha $=$ 0.933 , which indicates high reliability of the instrument. The findings from the heuristics questionnaire suggest that the game's assets of clarity, ease of use, appropriateness, quality, motivation, and mental effort were moderately favorable ( $>3$ ). Comments from experts propose that the game is causally fun, suitable for the target audience, and that the overall concept of the game is good with high potential.

Conclusion: WW could help many people who are fighting not just with cancer, but any disease, where physical activity is an important part of their recovery. Nonetheless, the treatment could be very exhausting for the patients. They must keep fighting as real warriors. Moreover, the game can be used by anyone who enjoys puzzle games or need motivation to walk.

Keywords: Serious game, Mobile game, HSCT patient, Physical activity, Motivation.

*Corresponding author. E-mail: lanyi@almos.unipannon.hu

\section{Experience Level and Usability Evaluation while Using "TATOO", a Touchscreen Assessment Tool} Alexandra Danial-Saad ${ }^{\mathrm{a}, *}$, Mattia Corzani ${ }^{\mathrm{b}}$ Carlo Tacconi $^{\mathrm{b}}$ and Lorenzo Chiari ${ }^{\mathrm{b}}$

${ }^{a}$ Occupational Therapy Department, Faculty of Social Welfare and Health Sciences, University of Haifa \& The Arab Academic College for Education in Israel, Haifa, Israel

${ }^{\mathrm{b}}$ Department of Electrical, Electronic, and Information Engineering University of Bologna, Bologna, Italy

Background: Touchscreen devices are widely used in modern life therapy sessions. A touchscreen assessment tool (TATOO) was developed by Daniel-Saad and Chiari to provide valid and reliable data regarding fine motor skills while operating these devices. This tool fits individuals of different ages and varying abilities. TATOO consists so far of six tasks, each of which provides information on a functional component required when using a touchscreen, such as a tap, swipe, pinch, drag, and so on. A numeric and graphic report summarizes the performance of each task includes temporal and Kinematic aspects, touch pressure and accuracy. TATOO was intended to be used by clinicians and by clients who are mostly without professional computer knowledge. For that reason, testing its usability is of paramount importance to predict the user's acceptance or rejection of the device and the degree of internal motivation to use it.

This paper reports a study that evaluated the usability of TATOO by both children with typical development and by clinicians. Furthermore, this paper shows the correlation between children's usability feedback and their level of experience to use touchscreen devices and also, the correlation between children's usability feedback and clinician's usability scores.

Method: Ten clinicians and 60 typical development children aged 4-10 have used this system in order to assess the usability of the tool. Clinicians were invited to the University of Haifa and given a three-hour presentation introducing TATOO and how to use it. Each of the clinicians was asked to assess six children using the TATOO application. After using TATOO, children evaluated their experience through the ShortFeedback-Questionnaire-Child (SFQ-Child), in which emoticons are used to describe the experienced level of difficulty. Usability Evaluation Questionnaire (SUS) and Semi-structured Interview were finally given to the clinicians. A demographic questionnaire was developed to gather data about the users.

Key results: The SFQ-Child questionnaire reached very high indices of pleasure (SFQ $=4.8$ of $5 \pm 0.49$ ), comfort ( $\mathrm{SFQ}=4.65$ of $5 \pm 0.92$ ), the sense of success $(\mathrm{SFQ}=4.9$ of $5 \pm 0.53)$, and ease of use (SFQ $=4.7$ of $5 \pm 0.38)$. Clinicians rated TATOO's usability as "very good" (SUS $=85.5 \pm 8.04$, range $=70-97.5$ ), which reflected their satisfaction with the system's usability. We found no correlation between the clinician usability scores and the SFQ-Child, which means that the clinician usability did not affect the children's experience. Furthermore, there is no correlation between SFQ-Child and the variable "experience using tablet or smartphone" in the demographic questions, which means that children's experience did not affect their usability feedback.

Conclusion: TATOO will complement the $21^{\text {st }}$ century assessment needs, where the traditional fine motor assessment tools are not able to capture the skills necessary to operate a touch screen device. The positive usability responses indicate that the new application has user-friendly characteristics and will likely have a successful use among the end users. Future studies with larger samples in different ages and with varying abilities are needed in order to update a new version with the universal design method.

Keywords: Touchscreen Assessment Tool (TATOO), Usability Evaluation, Short-Feedback-QuestionnaireChild. 
*Corresponding author. E-mail: saadalexandra@ gmail.com

\section{A Walking Assist Robot Which makes the Pelvis Move More Easily and Assists in Walking \\ Masataka Ootani ${ }^{\mathrm{a}}$, Atsushi Nakahara $^{\mathrm{a}}$, Hiroshi Tsutsui $^{\mathrm{a}}$, Kenji Yamada ${ }^{\mathrm{b}}$, Jun Kurihara ${ }^{\mathrm{c}}$ and Yukio Honda $^{\mathrm{a}, *}$ \\ ${ }^{a}$ Robotics Department, Osaka Institute of Technology, 1-45, Chayamachi, Kita-ku, Osaka, 530-8568, Japan \\ ${ }^{\mathrm{b}}$ Department of Electrical Systems Engineering, $\mathrm{Hi}$ - roshima Institute of Technology, 2-1-1 Miyake, Saeki- ku, Hiroshima 731-5193,Japan \\ ${ }^{\mathrm{c}}$ The Canon Institute for Global Studies, 1-5-1 Marunouchi,Chiyoda-ku,100-6511 Tokyo, Japan}

Background: One of the problems of the aged society is an increase in elderly people who have weakened muscle power. The most effective exercise therapy to counter physical frailty is walking accompanied by aerobic exercise. The walking assist robot in this research is designed not to rehabilitate frailty but to mitigate frailty for those elders who can walk by themselves. In other words, this walking assist robot does not improve the muscular strength of the leg, but is a robot that enables the person to walk faster.

Method: Our idea is that the walking assist robot can make the pelvis rotate more smoothly and to stimulate the sacrum which in turn enables a faster walking with a longer stride. Since the sacrum is located in the center of gravity in the human body, the robot makes it easier to rotate the pelvis by stimulating the right and left of the sacrum alternately according to the timing of the swing of the legs. The walking assist robot is composed of a pushing mechanism to stimulate the sacrum having one motor. When the pre swing stage of the lower limb is detected by the acceleration sensor attached to the thigh, a pushing stimulus is given to the right and left of the sacrum. The walking experiment was carried out in an $11 \mathrm{~m}$ length walking path by using a motion capture camera. We also conducted a SUS (System Usability Scale) questionnaire after the experiments. For this preliminary study, five healthy male student subjects were selected and measurements were done under the approval of the ethics committee of Osaka Institute of Technology.

Key results: Among the three prospective distances, i.e. $5 \mathrm{~mm}, 10 \mathrm{~mm}$, and $15 \mathrm{~mm}$, the best result was obtained in the case of the $15 \mathrm{~mm}$ distance. The highest performance for the timing of stimulation was ob- served at a time when the motor drive started stimulating while the leg was between pre swing and middle swing. As a result of the measurements using the motion capture, by increasing the extrusion amount of pressing stimulus, 3 of 5 showed an increase in walking speed, stride, pelvic rotation angle and the hip joint rotation angle. In the SUS questionnaire result of 6 items, the average was 59.5 points, which presents a problem concerning the complexity of the device.

Conclusion: It was our intention to develop a walking assist robot which would make the pelvis move more easily and assist people in walking. This robot will assist a person to walk more quickly using his or her own muscular strength without changing the strength of the person's own muscles. We believe we have introduced a novel means of improving pelvic rotation by pushing stimulation of the sacrum. As a result of preliminary experiments using healthy subjects, we hope that the effectiveness of this walking assist robot will lessen the effect of physical frailty for people in the aging population.

Keywords: Walking assist, Sarcopenia, Locomotive syndrome, Rotation of the Pelvis, Stimulating the sacrum

*Corresponding author. E-mail: yukio.honda@ oit.ac.jp

VIVO-Rehab: Coupling Humanoid Robots with Motion Sensing Devices to Support Upper Limb Function Assessment of Children with Spinal Muscular Atrophy (SMA)

Chiara Lepore ${ }^{\mathrm{a}}$, Lisa Cesario $^{\mathrm{a}}$, Massimiliano Malavasi $^{\mathrm{a}}$, Elisabetta Dondi ${ }^{\mathrm{a}}$, Lorenzo Desideri ${ }^{\mathrm{a}, *}$, Annarosa Colonna $^{\mathrm{b}}$ and Antonella Cersosimo ${ }^{\mathrm{b}}$

a AIAS Bologna onlus, Piazza della Pace, Piazza della Pace 4/A, 40134 Bologna, Italy

${ }^{\mathrm{b}}$ MEDICINA RIABILITATIVA INFANTILE IRCCS Istituto delle Scienze Neurologiche di Bologna, Via Sant'Isaia 90, 40123, Bologna, Italy

Background: Emerging off-the-shelf technologies such as motion sensing input devices and social robots are increasingly used as assistive technologies (AT) not only to compensate for people's disabilities, but also to support health professionals in their daily activities in order to develop more enjoyable rehabilitation scenarios. In this contribution we report on the development of VIVO-Rehab, a platform for the supervision, monitoring, assistance and rehabilitation of users from their homes. VIVO-Rehab was pilot-tested involving three children with Spinal Muscular Atrophy (SMA) to doc- 
ument the applicability of the platform in the evaluation of children's upper limb functions.

Method: VIVO-Rehab was designed to monitor preestablished parameters such as the analysis of movement for rehabilitation through the control of kinematic, dynamic and other physiological signals. The system integrates the Kinect device by Microsoft for Motion Capture and the humanoid robot NAO by Softbank Robotics. The NAO robot was used to instruct and demonstrate exercises. In this view, a set of predefined upper-limb movements was developed in collaboration with rehabilitation professionals involving elbow and shoulder joint movements. The set of upperlimb movements was then pilot tested with three children with SMA3 and SMA2 who were asked to perform movements shown by the Nao robot. During child-robot interactions, the Kinect recorded the data of the 25 joints. Subsequently these data were implemented in a Matlab program that allows the visualization of joints and segments during movement and allows the extraction of some parameters such as speed, acceleration and angles.

Key results: Observational results from the pilot study show that the use of Nao robot allows a greater interaction with children. Furthermore, the use of the Kinect allows to obtain good performances for the Motion Capture and with the Matlab program it was possible to perform both motion evaluations and the comparison between the movements.

Conclusion: This system can be applied both in the home and in the outpatient setting to perform continuous evaluations over time and monitor the improvement or deterioration of patients' movements. Future prospects see these devices implemented at home, useful for greater monitoring or home rehabilitation, so that they can easily see the results of the exercises performed or the correctness of the movements.

Keywords: movement detection, SMA, rehabilitation *Corresponding author. E-mail: ldesideri@ausilioteca. org

\section{Socially Assistive Robots Influence for Elderly with Cognitive Impairment Living in Nursing Facilities: Micro Observation and Analysis}

Toshiharu Igarashi $^{\mathrm{a}}$, Misato Nihei ${ }^{\mathrm{a}, \mathrm{b}, *}, \quad$ Mio Nakamura $^{\mathrm{b}}$, Kazuko Obayashic, ${ }^{\mathrm{c}, \mathrm{e}}$, Shigeru Masuyama $^{\mathrm{d}, \mathrm{f}}$ and Minoru Kamata ${ }^{\mathrm{a}, \mathrm{b}}$

${ }^{a}$ Department of Human and Engineered Environmental Studies, The University of Tokyo, 5-1-5 Kashiwanoha, Kashiwa, Chiba, Japan
${ }^{\mathrm{b}}$ Institute of Gerontology, The University of Tokyo, 51-5 Kashiwanoha, Kashiwa, Chiba, Japan

${ }^{\mathrm{c}}$ Social Welfare Corporation Tokyo Seishin-kai, 2-1625, Mukoudaicho, Nishitokyo, Tokyo, Japan

${ }^{\mathrm{d}}$ Universal Accessibility Evaluation Organization, 224-28, Hyakunincho, Shinjyuku, Tokyo, Japan

${ }^{\mathrm{e}}$ Faculty of Healthcare Management, Nihon Fukushi University, Mihama, Chitagun, Aichi Japan

${ }^{\mathrm{f}}$ Travellers Medical Center, Tokyo Medical University, 6-7-1 Nishishinjyuku, Shinjyuku, Tokyo, Japan

Background: In recent times, the lifestyle of the elderly in Japan has changed considerably. While previously two or three households lived together, in general, now there is an increase in the number of lonely and elderly couple households. Furthermore, according to a Japanese government report, the number of elderly people with dementia, which was 4.62 million in 2012, is estimated to increase to 7.3 million by 2025 . In this social context, using assistive technology to support the lives of the elderly can help them become more independent.

Method: Therefore, to observe and analyze the influence of social assistive robots on elderly people and nursing workers in a nursing care facility, a long-term intervention experiment was conducted from February 5 to February 25, 2018 (35 days), on ten elderly persons. The subjects' ages ranged from 81 to 97 years (Mean $=89.4, \mathrm{SD}=4.81$ ). All the subjects (one male, nine female) could express their willingness to participate in the study, including those with mild cognitive impairment.

Key results: The findings showed that the number of dialogues and dialogue time vary depending on their cognitive function. While the average intervention was 6.4 the 1 st week, 13.0 the 2 nd week, and 17.0 the 3 rd week in the group whose CDR $\leqq 1$, the average intervention was $2.6,7.2$, and 12.8 in the $1 \mathrm{st}, 2 \mathrm{nd}$, and $3 \mathrm{rd}$ week, respectively, in the group with $\mathrm{CDR} \geqq 2$. There were significant differences between the two groups $(p<0.05)$. However, the average number of dialogue times increased gradually in both groups in the intervention period. Also, in the conversation content analysis, it was found that the group with CDR $\leqq 1$ used more different kinds of words than the group with CDR $\geqq 2$. In addition, the average interaction time was 439.4 $\mathrm{sec} /$ week for the group with CDR $\leqq 1$, whereas it was $148.6 \mathrm{sec} /$ week for the group with $\mathrm{CDR} \geqq 2$. There was a significant difference between the two $(p<0.05)$. However, in the group with CDR $\leqq 1$, the average daily conversation time/week was almost the same, whereas it gradually increased in the group with $\mathrm{CDR} \geqq 2$. 
Conclusion: This could be attributed to the sound quality, pitch, and information presentation method of assistive robots using synthesized speech, making it difficult for them to be understood by elderly people with cognitive impairment. Therefore, correlating the frequency of artificial speech voice and degree of comprehension and cognitive levels is a future research subject.

Keywords: Socially assistive robots, Assistive technology, Elderly care, Human Computer Interaction, Sota,.

Acknowledgement: This research was supported by AMED (Agency for Medical Research and Development). This research was approved by the Research Ethics Committee of the University of Tokyo and Social Welfare Corporation Tokyo Seishin-kai.

*Corresponding author. E-mail: mnihei@edu.k.utokyo.ac.jp

\section{A Trial of a Communication Robot at Home for El- derly Living Alone Using Data from a telephone call beginning with "How Are You?"}

Satoshi Tsujikawa ${ }^{a}$, Kazuhira Akamatsu $^{\mathrm{b}}$, Hiromitsu Yokoyama $^{\mathrm{b}}$, Jun Kurihara ${ }^{\mathrm{c}}$ and Yukio Honda ${ }^{\mathrm{b}, *}$

${ }^{a}$ Tsujikawa ENT Clinic, 40-8 Ebata-cho, Kadoma, 571-0012 Osaka, Japan

${ }^{\mathrm{b}}$ Osaka Institute of Technology, 1-45 Chayamachi, Kita-ku, 530-8568 Osaka, Japan

${ }^{\mathrm{c}}$ The Canon Institute for Global Studies, 1-5-1 Marunouchi, Chiyoda-ku, 100-6511 Tokyo, Japan

Background: The declining birthrate and longevity have become pressing issues in various countries including Japan. Effective sharing of health information regarding healthy elderly living alone is expected to extend healthy longevity by their own preventive health management, leading to significant improvement of the sustainability of health and economical systems. We have made calls with 168 elderly living alone for 8 years at regular intervals and collected the information which could be of use (CALL data), and supported them by sharing the information. To continue this activity, we planned a communication robot with the Question-Answer-Response format(QAR) via buttons. The aim of this study is to determine whether the robot can communicate with elderly people with fixed QAR without using the pattern matching technology.

Method: Using CALL data, the subjects to be discussed after many conversations was narrowed down to 30 items and 979 QAR were made and classified into three categories, namely, mundane topics (366 QAR), physical topics (291 QAR) and mental topics (322 QAR). We adopted a push button answering system for elders with feeble verbal communication due to aging and/or disease. These questions include ones related to day-to-day life as mundane topics, e.g. "How are you?", being asked at first, and then, problems associated with physical and/or mental topics, if any. Topics that they hoped for were investigated by repeated inquiries via buttons and thereupon advice could be provided. We conducted this two-way communication with general mundane issues at first, and then with specific ones by repeating QAR. Since the Profile of Mood States 2nd Edition and Short Form is used worldwide to estimate the psychological condition, our experiment is evaluated using POMS2-Adult Short. 13 participants (Average age:79.3) of elderly living alone (9/13 were targeted for "How are you?" calls) and tried the robot at home on a one-to-two-month interval, and the abovementioned information was examined to evaluate psychological effects in pre- and post-trial, and the willingness to possibly extend at the end of the examination. Tension-Anxiety (TA), Depression-Dejection (DD), Anger-Hostility (AH), Vigor-Activity (VA), FatigueInertia(FI) and Confusion-Bewilderment(CB) were measured before and after the experiment. The rate of the improvement of the mood status was performed using paired t-study by TMD (Total Mood Disturbance $(\mathrm{TA}+\mathrm{DD}+\mathrm{AH}+\mathrm{FI}+\mathrm{CB})-\mathrm{VA})$. Furthermore, their intention to extend use was investigated at the end of the experiment.

Key results: 13 participants showed statistically significant improvement in mood status during the experiment and 9 participants wanted an extention of the trial period. Furthermore, 13 participants were divided into two groups on the basis of the mean value of total TMD percent improvements: good improvement group (7 participants) and insufficient group (6 participants). The average age (Mean $\pm \mathrm{SD}: 83.3 \pm 3.9$ ) in the Good Group was significantly older compared with that of the insufficient Group (Mean \pm SD: $75.2 \pm 3.9$ ).

Conclusion: The past eight years of our experiment have demonstrated that we can clearly identify the condition of the elderly living alone by asking only thirty questions with the help of our experimental communication robots.

Keywords: Communication Robot, Elderly Living Alone, Q\&A Format, Chatbot, Tele-counseling database

*Corresponding author. E-mail: yukio.honda@oit.ac.jp 